\title{
Thermal and Dynamic Mechanical Analyses of Poly(Lactic Acid)/Poly(Ethylene Glycol) Blends
}

\author{
Aouachria Kamira, *Benaniba Mohamed Tahar
}

Laboratoire des Matériaux Polymériques MultiPhasiques, Faculté de Technologie, Université Ferhat ABBAS, Sétif 1

Sétif 19000, Algérie.

\begin{abstract}
:
Blends of poly(lactic acid) (PLA) and poly(ethylene glycol) (PEG) with various contents $(0,5,10,15$, 20 and 30 weight \%) and with different molecular weights $\left(\overline{\mathrm{M}}_{\mathrm{w}}=1000,4000\right.$ and $\left.6000 \mathrm{~g} / \mathrm{mol}\right)$, called respectively PEG1, PEG2, and PEG3 were prepared by melt blending. Since glass transition temperature $(\mathrm{Tg}), \mathrm{T} \alpha$ and loss factor $(\tan \delta)$ are relevant indicators of polymer chain mobility, plasticization has been studied by dynamic mechanical analysis (DMA) and differential scanning calorimetry (DSC). Low molecular weight (LMW) PEG enable increased miscibility with PLA and more efficient reduction of glass transition temperature (Tg) for concentrations of PEG less than $20 \%$. This effect is not only enhanced by the LMW but also by increasing its content up to $20 \%$. As expected, both T $\alpha$ and Tg decrease when increasing PEG molar mass and content up to $20 \%$, which demonstrates the effectiveness of PEG to act as a plasticizer of PLA.
\end{abstract}

Key words: PLA, PEG, Plasticization, DSC, DMA.

\section{Introduction}

Poly(lactic acid) (PLA) is a biodegradable, compostable, biocompatible polymer used in food contact applications. This linear aliphatic thermoplastic polyester, derived from renewable resources, is decomposed without environmental pollution [1-3].

In order to improve the flexibility of PLA, it can be mixed with other polymers such as linear low density polyethylene [4-5], poly(vinyl acetate) [6], poly(ethylene glycol) [7], poly( $\varepsilon$ caprolactone) [8], poly(hydroxy butyrate) [9], and poly(hexamethylene succinate) [10]. Possible plasticisers for PLA include oligomeric lactic acid, lactide as well as low molecular weight esters such as citrates [11-12].

Poly (ethylene glycol) (PEG) is also biodegradable, has good miscibility to be used in PLA plasticization [13]. Jacobsen and Fritz [14] studied the plasticization of PLA by comparing the type and content of three plasticizers: poly(ethylene glycol), glucose monoesters, and fatty acid esters. By investigating the mechanical properties, the results show that PEG has good plasticizing effect on PLA.

Polyethylene glycols (PEG), also known as macrogels, are liquid or solid polymers of general formula $\mathrm{H}\left(\mathrm{OCH}_{2} \mathrm{CH}_{2}\right)_{n} \mathrm{OH}$. Low molecular weight $\mathrm{PEG}$ is in the liquid form, whereas higher molecular weight PEG are in the solid form at room temperature. PEG are suitable plasticizers for PLA because of their miscibility, biodegradability, and food contact applications. As most of the researchers use high molecular weight PEG as plasticizers, there is about no literature examining the plasticizing effect of low molecular weight PEG on PLA [15-16].

*Corresponding author: Benaniba Mohamed Tahar, Laboratoire des Matériaux Polymériques MultiPhasiques, Faculté de Technologie, Université Ferhat ABBAS, Sétif 1, Sétif 19000, Algérie.

E-mail address: m benaniba@yahoo.com, m benaniba@univ-setif.dz, Phone: +213777321252 
Given that, the influence of molecular weight on the thermal properties of PLA/PEG blends has not been reported yet in the literature. Consequently, in the present work, PEGs with different molecular weights were used as plasticizers of PLA. The effects of both content and molecular weight of PEG on the glass transition temperature of PLA/PEG blends were investigated.

\section{Materials and Method}

\subsection{Materials.}

PLA (2002D) was provided by Nature Works LLC (USA). Its density, melting point (Tm) and glass transition temperature $(\mathrm{Tg})$ are $1.20 \mathrm{~g} / \mathrm{cm}^{3}, 170$ and $60^{\circ} \mathrm{C}$ respectively. The PEGs with three different molecular weights are used as plasticizers of PLA. The PEG were supplied by Pancrea Chemical Reagent Co., Ltd (spain). Their average molecular weights are 1000, 4000 and 6000. They are called PEG1, PEG2 and PEG3, respectively.

\subsection{Process and Formulations}

Before processing by melt blending, PLA and PEGs were dried under vacuum, respectively at $70^{\circ} \mathrm{C}$ for 5 hours and at $50^{\circ} \mathrm{C}$ for 8 hours. Then, the PLA pellets were respectively premixed with PEG. The mixtures of PLA/PEG were melt blended at $190^{\circ} \mathrm{C}$ for 12 minutes at $40 \mathrm{rpm}$ using Poly Lab Torque Rheometer (Haake Instrument Corp. Germany). The mixing ratios of the PLA/PEG blends were $95 / 5,90 / 10,85 / 15,80 / 20$, and 70/30 wt/wt.

The PLA pellets were also processed under identical processing. The PLA and PLA/PEG blends were cut into granules and dried again. The blends obtained were then molded into $1 \mathrm{~mm}$ thick sheets by hot pressing at $190^{\circ} \mathrm{C}$ for 4 minutes with pressure of $110 \mathrm{~kg} / \mathrm{cm}^{2}$, followed by cooling at room temperature. Plasticized PLA with contents $n$ of PEG are named PLA-PEG-n.

\subsection{Differential scanning calorimetry (DSC)}

Thermograms were obtained from a TA instrument differential scanning calorimeter (DSC) Q10. The DSC procedure was composed of three steps. In the first step, the samples were heated from $30^{\circ} \mathrm{C}$ to $220^{\circ} \mathrm{C}$ with a heating rate of $20^{\circ} \mathrm{C} / \mathrm{min}$. Then, they were held at this temperature for 5 min to eliminate the thermal history, cooled to $-50^{\circ} \mathrm{C}$ at a cooling rate of $20^{\circ} \mathrm{C} / \mathrm{min}$ and held at $50^{\circ} \mathrm{C}$ for $5 \mathrm{~min}$. In the last step, they were reheated to $220^{\circ} \mathrm{C}$ at a heating rate of $10^{\circ} \mathrm{C}$. All the temperatures (Tcc, Tm) measured at the peak maximum were determined with an accuracy of less than $0.5^{\circ} \mathrm{C}$. Crystallization and melting temperatures, respectively Tcc and Tm, as well as $\mathrm{Tg}$, were determined. The crystallinity $(\mathrm{Xc})$ of all samples was calculated according to :

$$
\mathrm{X}_{\mathrm{c}, \mathrm{PLA}}(\%)=\frac{\Delta \mathrm{H}_{\mathrm{m}, \mathrm{PLA}}-\Delta \mathrm{H}_{\mathrm{cc}, \mathrm{PLA}}}{\Delta \mathrm{H}^{0}{ }_{\mathrm{m}, \mathrm{PLA}} \mathrm{X}_{\mathrm{PLA}}} \times 100
$$

Where $\Delta \mathrm{H}_{\mathrm{m}, \mathrm{PLA}}$ is the measured heat of fusion, $\Delta \mathrm{Hcc}$ is the heat of cold crystallisation, $\mathrm{X}_{\mathrm{PLA}}$ is the PLA content in the blend and $\Delta \mathrm{H}^{0}$ m,PLA is the melting enthalpy of PLA that would be $100 \%$ cristalline $(93 \mathrm{~J} / \mathrm{g})$.

\subsection{Dynamic mechanical analysis (DMA)}

The dynamic mechanical properties of the plasticised PLA were carried out using a dynamic mechanical analyzer (RSA) in tensile mode. The measurements were performed at a constant frequency of $1 \mathrm{~Hz}$ in the temperature range $\left[20^{\circ} \mathrm{C}, 100^{\circ} \mathrm{C}\right]$ at a heating rate of $3^{\circ} \mathrm{C} / \mathrm{min}$. 


\subsection{Thermogravimetric analysis (TGA)}

TGA was carried out using a TA instrument Q500 with scans from $35^{\circ} \mathrm{C}$ to $500^{\circ} \mathrm{C}$ at a constant heating rate of $10^{\circ} \mathrm{C} / \mathrm{min}$, under a continuous nitrogen flow. The thermal degradation temperatures taken into account were the onset temperature (Tonset), $\mathrm{T}_{5}$ and $\mathrm{T}_{50}$ which are respectively the temperature at 5 and $50 \%$ weight loss, and the temperature of maximum weight loss (Tmax).

\section{Results and discussion}

To further explain the miscibility and plasticizing effect of PEG with the PLA matrix, PLA/PEG blends with $0,5,10,15,20$, and $30 \%$ of PEG were prepared, to carry out DSC, which results are given on Figure 1. The DSC thermograms last heating scans of plasticized PLA with PEG1, PEG2, and PEG3 are displayed on Figure 1 (a), (b), and (c) respectively. Pure PLA has Tg around $60^{\circ} \mathrm{C}$ which shifts to low temperature when the PEG concentration increases up to $20 \%$, whatever its molar mass. Moreover, Tcc of the PLA matrix decrease when increasing the plasticiser concentrations up to $20 \%$, this is consistent with the fact that PLA crystallizes easier at lower temperatures because of the enhanced chain mobility as the plasticizer content increases.

(a)

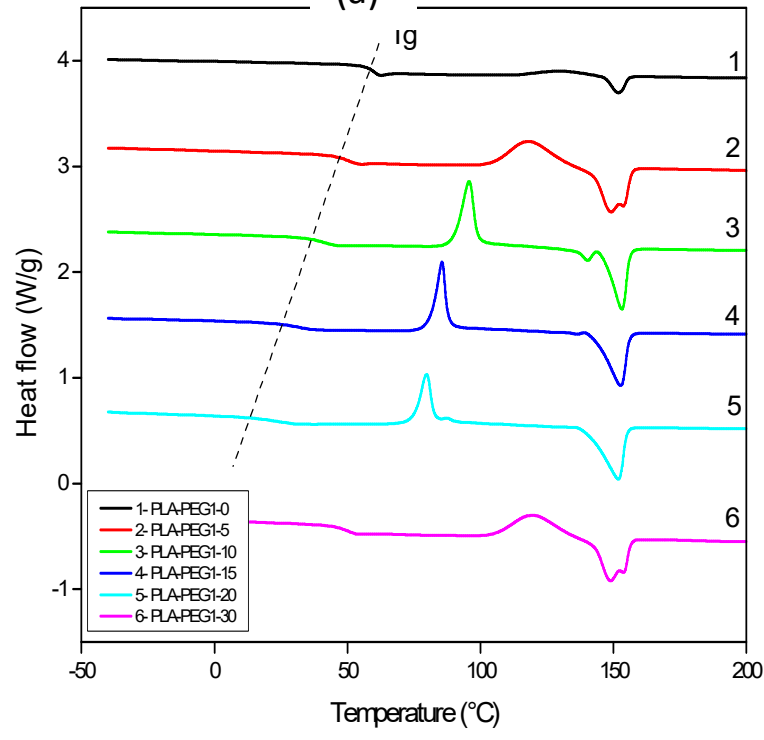

(b)

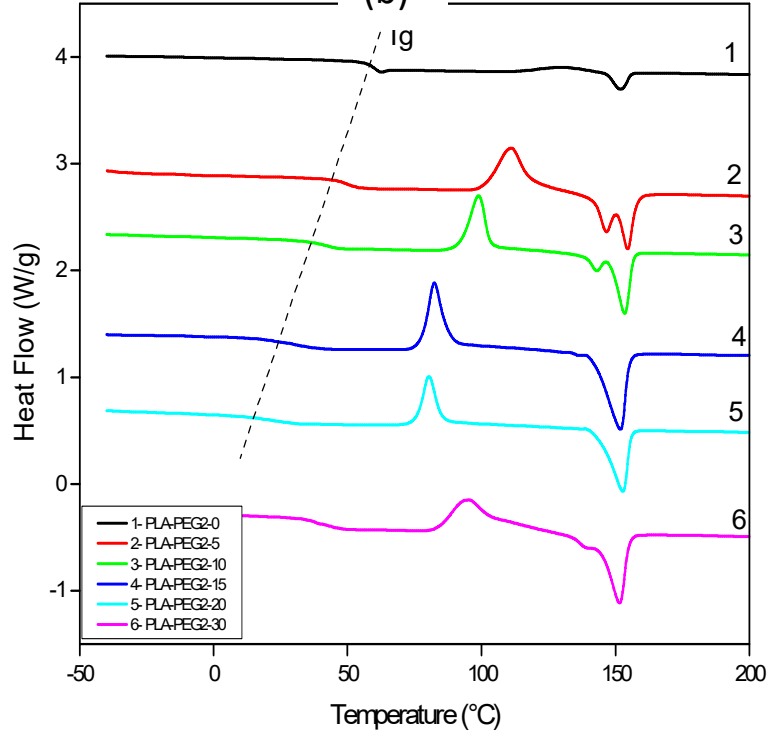


(c)



Figure 1. DSC thermograms of PLA/PEG at various PEG contents. (a) PEG1, (b) PEG2, (c) PEG3.

The plasticization of PLA, with concentrations of PEG up to $20 \%$, decreases glass transition temperatures (Tg) (Figure 2(a)) as well as cold crystallization temperatures (Tcc) (Figure 2(b)). Low molecular weight PEG1 is the most efficient to lower the intermolecular forces and increase the mobility of the polymer chains, thereby improving the flexibility by decreasing $\mathrm{Tg}$.

For contents higher than $20 \mathrm{w} \%$ of plasticiser, it is clear that all the blends present a limit of miscibility and $\mathrm{Tg}$ attains a plateau value. The crystallinity of the plasticized blends by PEG increase until $20 \%$, whatever the PEG used.

(a)

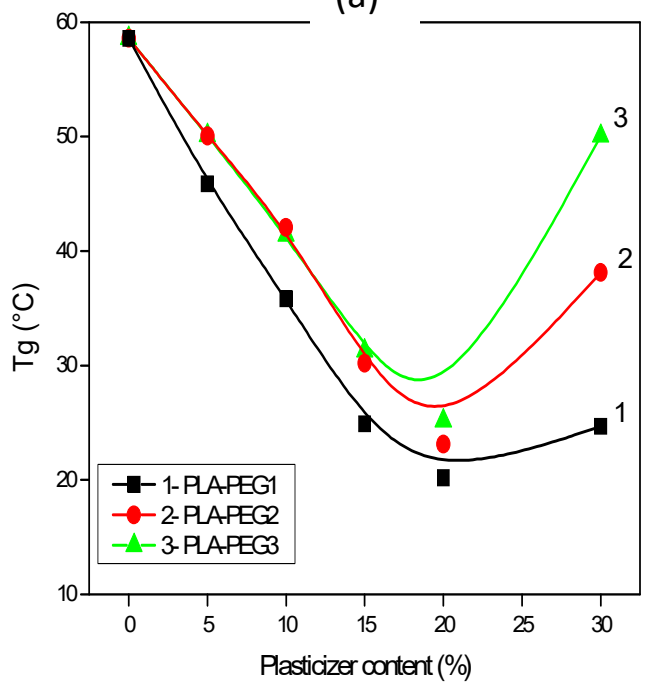

(b)

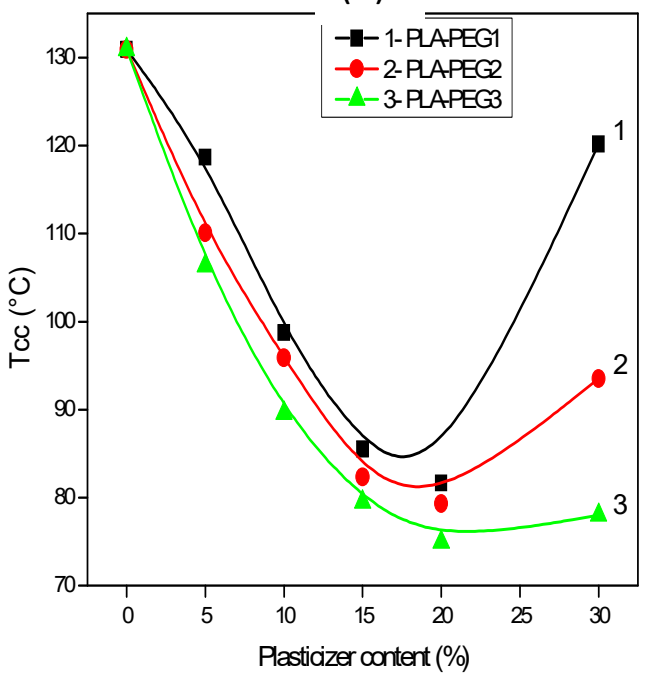


(c)

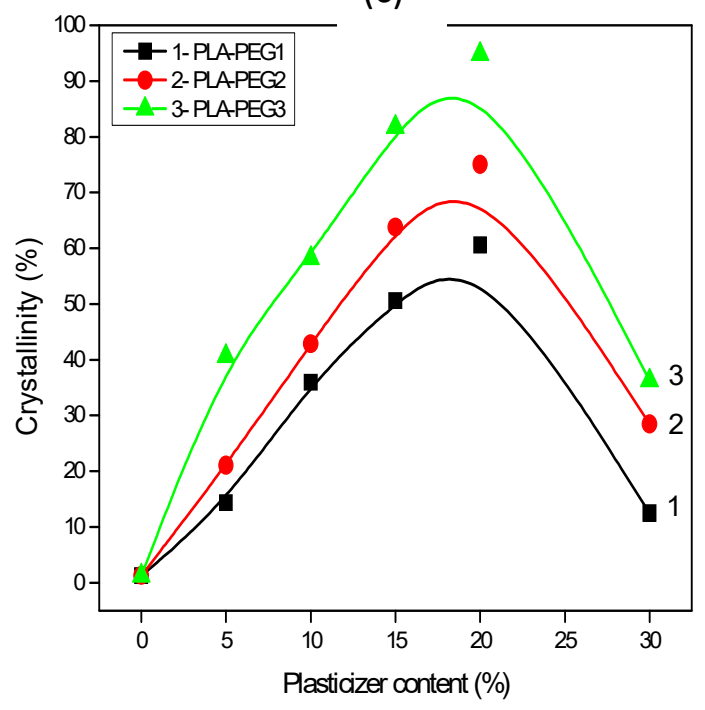

Figure 2. Glass transition temperature $\mathrm{Tg}$ (a), cold crystallization temperature (b), and crystallinity (c) of plasticized PLA at various PEG contents of PEG1, PEG2, and PEG3.

PLA can crystallize at higher temperatures than PEG. The addition of the PEG plasticizer decreases Tg and increases the crystallinity of blends as shown in Figure 2 (c). This is attributed to the enhanced motion of the PLA molecular chains. Plasticizers decrease Tg and make materials more flexible. Plasticizers interact with the PLA chains at molecular level, speed up the viscoelastic response and increase the mobility of chains [17].

We conclude that the plasticizing effect of PEG is improved when decreasing $\bar{M}_{w}$ of PEG. The same trend is shown on Figure 2 (a) and (b), where both Tg and Tcc values of the blends also shift to lower temperatures when decreasing $\overline{\mathrm{M}}_{\mathrm{w}}$ of PEG.

Dynamic mechanical analysis (DMA) as a function of temperature at a fixed frequency is a well known method to determine $\mathrm{T} \alpha$, which is a temperature associated to the glass transition temperature (Tg). Figure 3 (a), (b) and (c) present respectively the temperature dependence of E', E", and the $\alpha$-relaxation temperatures of the blends obtained by DMA. The decrease of storage modulus (E') and loss modulus (E") peak temperature is attributed to plasticization.

From Figure 3 (c), only a T $\alpha$ peak existing in every DMA curve is observed, which indicates that compatibility of the PLA and of three kinds of plasticizers is excellent. The DMA data indicate that the T $\alpha$ peaks shift to lower temperatures when increasing PEG content.

The DMA curves of PLA show that T $\alpha$ is higher than Tg detected by DSC. The decrease of Tg indicates that the chain mobility of PLA increases. The evolution of PLA's Tg is consistent with the common rule that plasticizers decrease Tg. In our study, PEG act as a plasticizers and increase the mobility of PLA chain segments. 
(a)

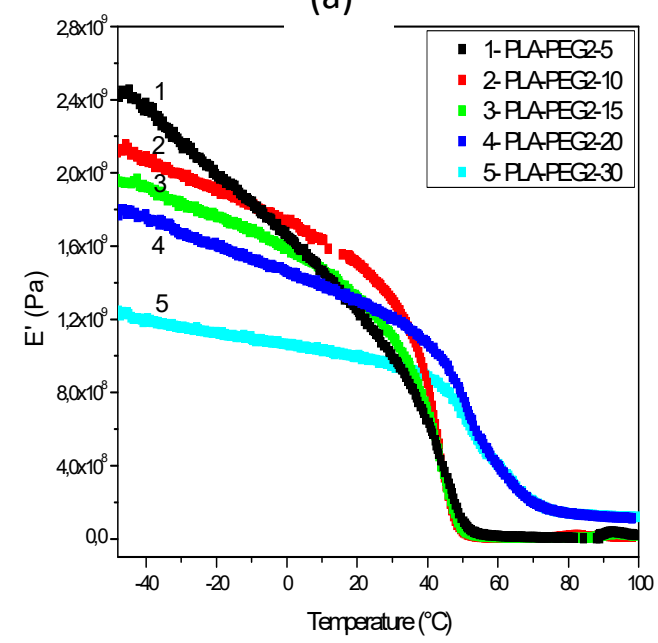

(b)

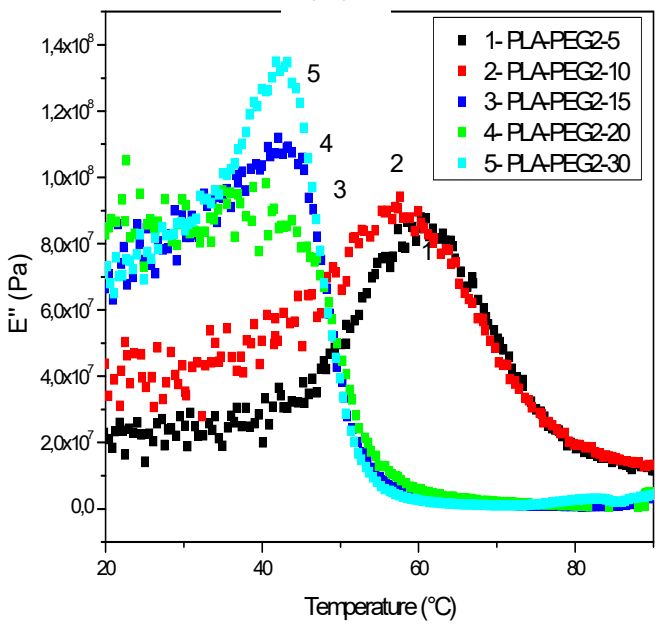

(c)

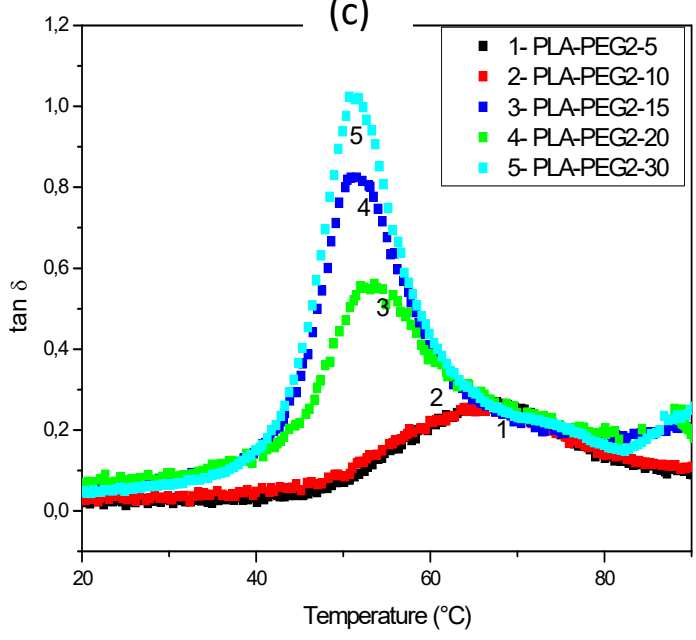

Figure 3. Dynamic mechanical behaviour (a), Storage modulus (b) Loss modulus, and (c) Loss factor of plasticized PLA with PEG2 samples.

Since the glass transition temperature $\mathrm{Tg}$ is an excellent indicator of polymer structure and chain mobility, plasticizing efficiency is therefore evaluated by the decrease in $\mathrm{Tg}$ as a function of the plasticizer concentration. In agreement with the evolution of Tg, Figure 4 shows that T $\alpha$ decrease from $64^{\circ} \mathrm{C}$ to $46^{\circ} \mathrm{C}$ when adding PEG. For contents higher than $20 \mathrm{w} \%$ of plasticizer it is clear that all the blends present a limit of miscibility and the glass transition temperature attains a plateau value. DMA demonstrates that the addition of PEG results in a decrease in T $\alpha$, and the reduction was the most effective with the PEG having the lowest molecular weight. 


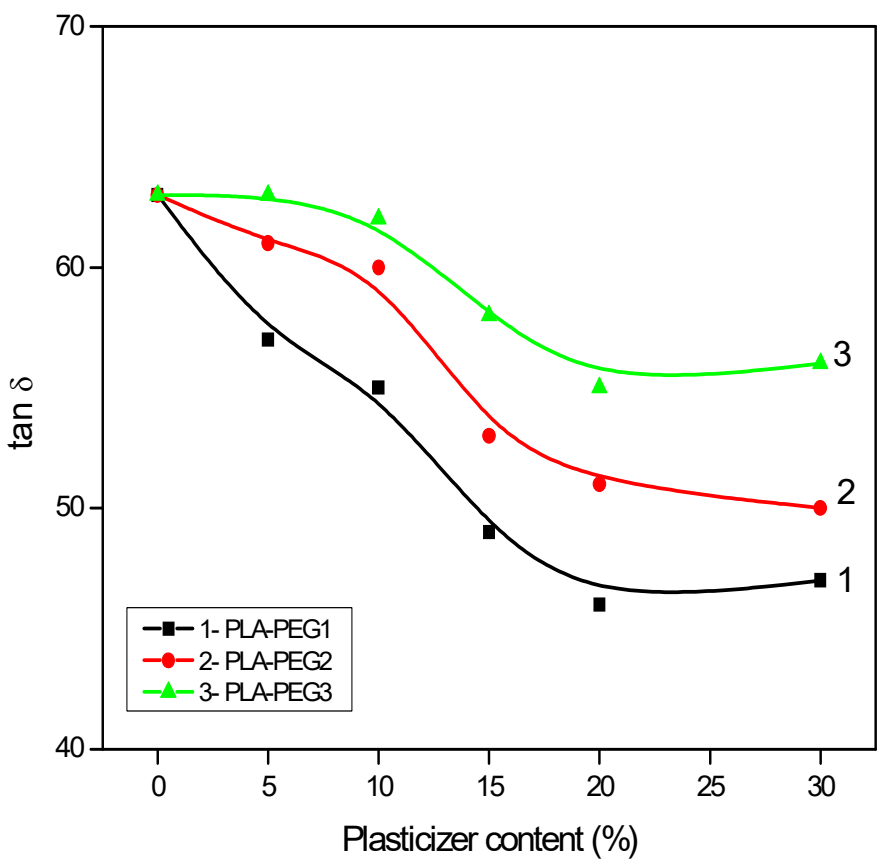

Figure 4. $\tan \delta$ of plasticized PLA at various contents of: 1- PEG1, 2- PEG2, 3- PEG3.

The DMA curve of PLA displays a marked drop in E' around $70^{\circ} \mathrm{C}$ (Figure 3 (a)) at a temperature slightly higher than the one of Tg value obtained by DSC.

Previous studies have shown that PEG blending with PLA presents different plasticization and miscible behaviors, which depend on the composition and molecular weight of PEG [18-20]. DMA experiments carried out by Baiardo et al.[21] reveal that the lower molecular weight PEG show better miscibility and plasticizing efficiency than the PLA matrix.

Thermal degradation of PLA and plasticized PLA are evaluated by thermal degradation analysis (TGA) and the corresponding derivative thermogravimetry analysis (DTG) curves are displayed in Figure $5(\mathrm{a}, \mathrm{b}$, and $\mathrm{c})$. $\mathrm{T}_{5 \%}$ and $\mathrm{T}_{50 \%}$ refer to the temperatures at which the remaining mass of the materials is $5 \%$ and $50 \%$ respectively (Table 1). Pure PLA is decomposed in a single step, which is characterised by a single peak at $394^{\circ} \mathrm{C}$ on DTG curves. The peak on DTG curve characterizes the onset decomposition temperature (Tonset), the maximal rate (Tmax) of the thermal degradation. The introduction of PEGs reduces the thermal stability of the PLA with a decrease in the Tonset and Tmax, in particular, for PLA with $20 \mathrm{wt} \%$ PEG.

$\mathrm{T}_{5} \%$ and $\mathrm{T}_{50 \%}$ of pure PLA are $346^{\circ} \mathrm{C}$ and $383^{\circ} \mathrm{C}$ respectively. It is revealed that the addition of PEG1 decreases these temperatures. The thermal stability of PLA increases with PEG molecular weight but decreases when increasing its content. This degradation behavior can be attributed to the plasticizing effect of PEG, which is expected to increase the relative movements of the PLA chains at lower temperatures. Nijenhuis et al [22] explain that the poor long-term stability of PLA/PEG blends is due to slow crystallization of PEG. Residual weights of all samples are lower than $2 \mathrm{wt} \%$ and no significant difference is observed between the samples. This shows that PEG does not promote char formation. 
Table 1. Data obtained from TGA and DTG thermograms of plasticized PLA-PEG.

\begin{tabular}{cccccc}
\hline \%PEG1 & Tonset $\left({ }^{\circ} \mathrm{C}\right)$ & $\mathrm{T}_{5 \%}\left({ }^{\circ} \mathrm{C}\right)$ & $\mathrm{T}_{50 \%}\left({ }^{\circ} \mathrm{C}\right)$ & $\mathrm{T}_{\max }\left({ }^{\circ} \mathrm{C}\right)$ & Residue (\%) \\
\hline 0 & 273.4 & 346.6 & 383.3 & 394.8 & 0.50 \\
5 & 250.4 & 341.7 & 387.4 & 396.2 & 0.70 \\
10 & 231.8 & 321.0 & 378.5 & 387.8 & 1.20 \\
15 & 242.9 & 326.7 & 379.4 & 387.3 & 1.10 \\
20 & 249.9 & 321.8 & 378.5 & 388.2 & 1.30 \\
30 & 232.3 & 312.8 & 373.5 & 382.6 & 1.20 \\
\hline$\%$ PEG2 & Tonset $\left({ }^{\circ} \mathrm{C}\right)$ & $\mathrm{T}_{5 \%}\left({ }^{\circ} \mathrm{C}\right)$ & $\mathrm{T}_{50 \%}\left({ }^{\circ} \mathrm{C}\right)$ & $\mathrm{T}_{\operatorname{mxx}}\left({ }^{\circ} \mathrm{C}\right)$ & Residue $(\%)$ \\
\hline 5 & 279.6 & 344.9 & 386.5 & 393.3 & 0.90 \\
10 & 289.7 & 345.5 & 387.7 & 394.7 & 0.90 \\
15 & 280.9 & 338.0 & 385.3 & 391.3 & 1.10 \\
20 & 271.7 & 333.2 & 384.9 & 391.8 & 1.30 \\
30 & 274.6 & 331.5 & 385.4 & 394.1 & 0.90 \\
\hline$\%$ PEG3 & Tonset $\left({ }^{\circ} \mathrm{C}\right)$ & $\mathrm{T}_{5 \%}\left({ }^{\circ} \mathrm{C}\right)$ & $\mathrm{T}_{50 \%}\left({ }^{\circ} \mathrm{C}\right)$ & $\mathrm{T}_{\max }\left({ }^{\circ} \mathrm{C}\right)$ & Residue $(\%)$ \\
\hline 5 & 262.5 & 348.4 & 387.4 & 393.6 & 0.90 \\
10 & 281.8 & 345.0 & 385.6 & 391.3 & 1.00 \\
15 & 290.4 & 337.7 & 384.1 & 388.5 & 1.00 \\
20 & 286.8 & 335.8 & 385.3 & 391.1 & 1.10 \\
30 & 269.0 & 330.6 & 386.9 & 391.8 & 1.10 \\
\hline
\end{tabular}

(a)

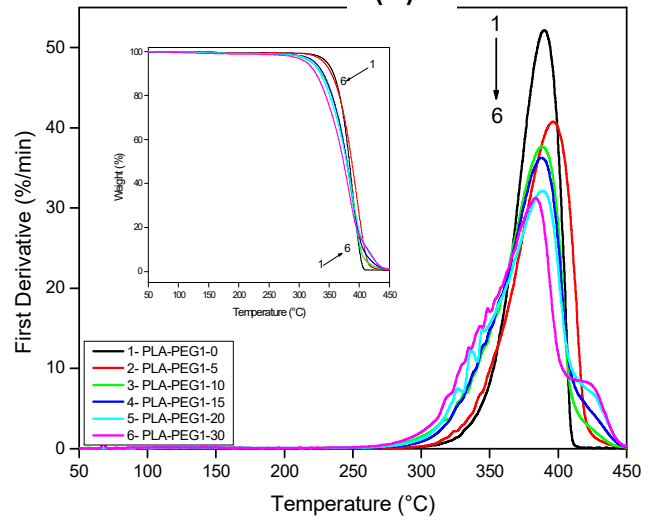

(b)

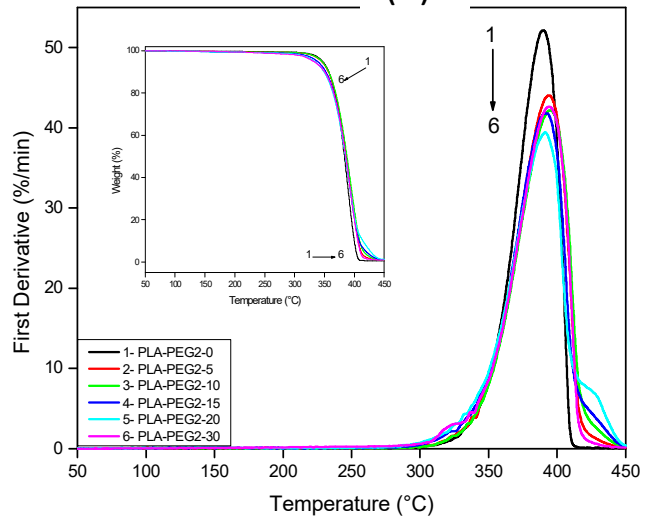

(c)

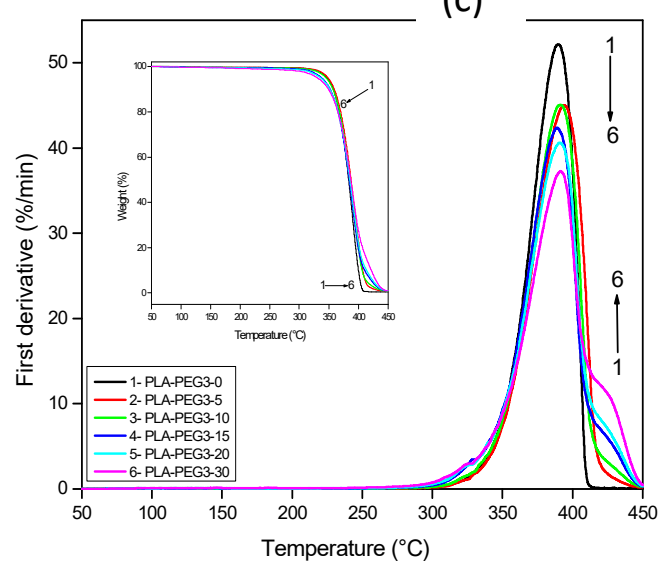

Figure 5. TGA and DTG thermograms of PLA/PEG at various PEG contents; (a) PEG1, (b) PEG2, (c) PEG3. 


\section{Conclusion}

The addition of PEG plasticizer to lower the glass transition temperature (Tg) and increase the crystallinity of the blends is attributed to the enhanced segmental motion of the PLA molecular chains.

PEGs blended with PLA decrease the glass transition temperature and modify the melting and crystallization characteristics. The PEGs are the most efficient for both $\mathrm{T} \alpha$ and $\mathrm{Tg}$ reduction and it clearly appears that for compositions up to $20 \mathrm{w} \%$ of plasticizer, all the blends present a limit of miscibility as the glass transition temperature reaches a plateau value.

The higher the weight percentage of PEG, the greater the plasticizing effect of the PLA matrix. The plasticizing effect is improved when decreasing the molecular weight.

Moreover, no volatilisation of plasticizer has been observed during melt compounding process. The improved thermal stability of the plasticizer in PLA blends could be due to the good affinity of PEGs with PLA. The results show that PLA/PEG blends with extent properties can be attained by optimizing the content and molecular weight of PEG.

\section{References}

[1] R. E. Drumright, P.R. Gruber, and D. E. Henton, "Polylactic acid technology," Advanced materials, vol. 12, no. 23, pp. 1841-1846, 2000.

[2] D. Garlotta, "A literature review of poly (lactic acid)," Journal of Polymers and the Environment, vol. 9, no. 2, pp 63-84, 2001.

[3] L. T. Lim, R. Auras, and M. Rubino, "Processing technologies for poly (lactic acid)," Progress in polymer science, vol. 33, no.8, pp. 820-852, 2008.

[4] K. S. Anderson, S. H. Lim, M. A. Hillmyer,"Toughening of polylactide by melt blending with linear low-density polyethylene,"Journal of Applied Polymer, vol. 89, no.14, pp. 3757-3768, 2003.

[5] B. Boubekeur, N. Belhaneche-Bensemra, and V. Massardier,"Valorization of waste jute fibers in developing low-density polyethylene/poly lactic acid bio-based composites," Journal of Reinforced Plastics and Composites, vol. 34, no. 8, pp. 649-661, 2015.

[6] A. M. Garjria, V. Dave, R. A. Gross, and S. P. McCarthy, "Miscibility and biodegradability of blends of poly(lactic acid) and poly(vinyl acetate)," Polymer, vol. 37, no. 3, pp. 437-444, 1996.

[7] M. Sheth, R. A. Kumar, V. Dave, R. A. Gross, and S. P. McCarthy, "Biodegradable polymer blends of poly (lactic acid) and poly (ethylene glycol),"Journal of Applied Polymer Science, vol. 66, no.8, pp. 1495-1505, 1997.

[8] Y. Cha, and C. G. Pitt, "The biodegradability of polyester blends," Biomaterials, vol. 11, no. 2, pp. 108-112, 1990.

[9] L. L. Zhang, C. D. Xiong, and X. M. Deng,"Miscibility, crystallization and morphology of poly ( $\beta$-hydroxybutyrate)/poly (d, l-lactide) blends," Polymer, vol. 37, no. 2, pp. 235-241, 1996.

[10] M. R. Lostocco, and S. J. Huang,"Aliphatic polyester blends based upon poly (lactic acid) and oligomeric poly (hexamethylene succinate)," Journal of Macromolecular Science, Part A Pure and Applied Chemistry, vol. 34, no. 11, pp. 2165-2175, 1997. 
[11] N. Ljungberg, and B. Wesslen, "The effects of plasticizers on the dynamic mechanical and thermal properties of poly(lactic acid)," Journal of Applied Polymer Science, vol. 86, no. 5, pp.1227-1234, 2002.

[12] M. Maiza, M. T. Benaniba, and V. Massardier,"Plasticizing effects of citrate esters on properties of poly (lactic acid)," Journal of Polymer Engineering, vol. 36, no. 4, pp. 371-380, 2016.

[13] J. Milton Harris,"Poly(ethylene glycol) chemistry: biotechnical and biomedical applications", Springer science Business Media, New York, 1992.

[14] S. Jacobsen, and H. G. Fritz,"Plasticizing polylactide - the effect of different plasticizers on the mechanical properties", Polymer Engineering \& Science, vol. 39, no.7, pp. 1303$1310,1999$.

[15] N. Stoehr, B; Baudrit, E. Haberstroh, M. Nase, P. Heidemeyer, and M. Bastian, "Properties and weldability of plasticized polylactic acid films," Journal of Applied Polymer Science, vol. 131, no. 12, pp. 40394-40403, 2014.

[16] F. J. Li, J. Z. Liang, S. D. Zhang, and B. Zhu, "Tensile Properties of Polylactide/Poly(ethylene glycol) Blends," Journal of Polymers and the Environment, vol. 23, no, 3, pp. 407-415, 2015.

[17] R. Song, R. Xue, H. He, Y. Liu, and Q. L. Xiao, "The structure and properties of chitosan/polyethylene glycol/silica ternary hybrid organic-inorganic films,"Chinese Journal of Polymer Science, vol. 26, no. 5, pp.621-630, 2008.

[18] I. Pillin, N. Montrelay, and Y. Grohens, "Thermo-mechanical characterization of plasticized PLA: Is the miscibility the only significant factor?"Polymer, vol.47, no.13, pp.4676-4682, 2006.

[19] Y. Hu, M. Rogunova, V. Topolkaraev, A. Hiltner, and E. Baer, "Aging of poly (lactide)/poly (ethylene glycol) blends. Part 1. Poly (lactide) with low stereoregularity," Polymer, vol. 44, no. 19, pp. 5701-5710, 2003.

[20] F. Yu, K. Prashantha, J. Soulestin, M. F. Lacrampe, and P. Krawczak, "Plasticizedstarch/poly (ethylene oxide) blends prepared by extrusion," Carbohydrate Polymers, vol. 91. No. 1, pp. 253-261, 2013.

[21] M. Baiardo, G. Frisoni, M. Scandola, M. Rimelen, D. Lips, K. Ruffieux, and E. J. Wintermantel, "Thermal and mechanical properties of plasticized poly (L-lactic acid)," Journal of Applied Polymer Science, vol. 90, no. 7, pp. 1731-1738, 2003.

[22] A. Nijenhuis, E. Colstee, D. W. Grijpma, and A. J. Pennings, "High molecular weight poly (L-lactide) and poly (ethylene oxide) blends: Thermal characterization and physical properties," Polymer, vol.37, no. 26, pp. 5849-5857, 1996. 